\title{
Le brûlage dirigé dans le Mercantour : savoirs pratiques, relations sociales et processus de décision
}

\author{
Ariane Bernard-Laurent ${ }^{\mathrm{a}}$, Florence Weber ${ }^{\mathrm{b}}$ \\ a Biologiste, Office national de la chasse et de la faune sauvage, CADAM, Préfecture est, 89 route de Grenoble, \\ 06286 Nice cedex 3, France \\ b Sociologue, École normale supérieure et Centre d'études de l'emploi, ENS, 48 boulevard Jourdan, 75014 Paris, France
}

\begin{abstract}
Afin d'éviter les développements non maîtrisables des incendies de forêt, une politique de brûlages dirigés est mise en œuvre en France depuis quelques années. Par la réouverture des milieux, elle vise ainsi également à contribuer à la préservation de la biodiversité et à la politique d'aide au pastoralisme. Cet article donne à voir comment cette politique est appliquée dans deux départements du Sud-est de la France et comment elle génère des procédures différenciées en fonction des configurations locales de chaque dispositif de mise en œuvre. Ainsi, dans une diversité de situations, selon les acteurs impliqués, les savoirs mobilisés, les traditions institutionnelles, l'intervention publique décentralisée peut déboucher sur des opérations totalement différentes avec un même objectif, domestiquer les feux de forêts.
\end{abstract}

La Rédaction

\section{Mots-clés :}

brûlage dirigé;

montagnes

méditerranéennes;

interdisciplinarité ;

méthode

ethnographique ;

gestion de

l'environnement

\section{Keywords:}

Prescribed-burning;

Mediterranean

mountains;

interdisciplinarity; ethnographic method; range management
Résumé - Après son institutionnalisation pour répondre au risque d'incendie lié à la déprise rurale, le brûlage dirigé est aujourd'hui requalifié comme technique d'ouverture du milieu, pour favoriser les activités pastorales et la biodiversité. Une enquête, réalisée dans le Mercantour en 2004-2005, met en évidence les spécificités de cette pratique dans chacun des deux départements du massif, les conditions de l'émergence d'un consensus entre praticiens de l'environnement et l'importance, pour la résolution d'un conflit local, des savoirs sociaux et relationnels et des rapports de force politiques à différentes échelles. Les auteurs, une sociologue utilisant la méthode ethnographique et une biologiste, préconisent des dispositifs de recherche interdisciplinaire pour étudier les objets de l'écologie et, dans le cas présenté ici, pour promouvoir une vision synthétique des zones susceptibles d'être réouvertes à l'échelle du massif du Mercantour.

\begin{abstract}
Prescribed-burning in Mercantour: practical knowledge, social relations and decision-processes. Prescribed-burning appeared in France in the 1980's in order to mitigate fire risk, which is itself related to rural abandonment and a calling into question of shepherds' collective skills. Another purpose of prescribed-burning is the opening of landscapes to favor grazing management and the maintenance of biodiversity. A survey undertaken in the Mercantour in 2004-2005 by a sociologist and a biologist, brings to the fore the institutional and social specificities of this practice in a mountain range located on two departments, the Alpes-Maritimes where burning is carried out by a corps of foresters-firemen, and the Alpes-de-Haute-Provence, where such a corps does not exist. The analysis of a conflict revolving around a prescribed-burning request, between a mayor and an intermittent resident, both hunters, and the study of the political and administrative modalities of its resolution, sheds light on the social conditions of emergence of a consensus between the different environmental practitioners, professionals and amateurs, and shows the importance of political power relations at different scales and of the different types of knowledge: practical knowledge on nature whose possessors benefit from a mutual recognition, without any consideration of professional status or school or administrative certificate, but also relational and social skills, related to the detailed knowledge of institutional procedures and local political issues. In conclusion, the authors recommend the drawing up of interdisciplinary research plans to study the objects of ecology and to question the absence of any synthetic view of areas that could be re-opened at the scale of the Mercantour range.
\end{abstract}

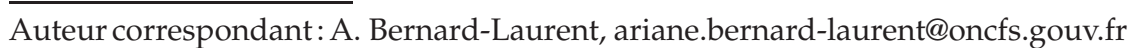


L'association d'une biologiste de l'Office national de la chasse et de la faune sauvage (ONCFS) et d'une sociologue au cours d'une enquête menée dans le Mercantour en 2004 et $2005^{1}$ (Tab. 1) a permis de mettre au centre des questions d'environnement, telles qu'elles se posent au quotidien, l'expérience des multiples protagonistes d'une microdécision : faut-il laisser en l'état telle portion du territoire communal embroussaillée depuis 50 ans ? Faut-il la brûler? Faut-il la débroussailler mécaniquement? La mutation du feu pastoral, pratique tolérée par l'État dans le cadre du droit de propriété depuis 1827, et dont les évolutions sont liées aux transformations du métier d'éleveur au XXe siècle, en brûlage dirigé, pratique contrôlée par des professionnels du feu et inscrite dans la loi en France en 2002, a été étudiée par des géographes (Métailié, 1981, 1998, 1999) et par des ethnologues (Ribet, 1996, 2000 ; Dumez, 2004). Notre étude examine les pratiques du brûlage dirigé comme le résultat d'un processus de décision où interviennent des acteurs pris dans des contextes différents et qui tiennent compte tout à la fois des enjeux de développement local, de sécurité publique, de gestion de l'environnement et de conservation de la nature (Buffière et al., 1992). Une réunion de concertation organisée dans un village des Alpes-Maritimes, après la contestation d'une demande de brûlage dirigé, entre des élus locaux et les services du conseil général et de l'ONF auxquels revient la décision, a donné l'occasion d'observer les relations in vivo entre des personnes mobilisées à titre privé par cette requête, éleveurs, propriétaires et chasseurs, et les protagonistes officiels de la décision : élus locaux et départementaux, services techniques départementaux et experts extérieurs. On insistera sur l'existence de deux types de savoirs complémentaires : des savoirs pratiques inégalement distribués et des savoirs relationnels, qui permettent à leurs détenteurs de tenir compte des rapports de force politiques à différentes échelles. Pour pouvoir analyser cette réunion, on rappellera d'abord le contexte politique, social et scientifique dans lequel se pratiquent aujourd'hui les opérations de brûlage dirigé, puis on mettra l'accent sur les spécificités du massif du Mercantour, qui relève de deux départements institutionnellement très différents, les Alpes-Maritimes et les Alpes-de-Haute-Provence. On s'interrogera, pour conclure, sur les effets du découpage départemental comme cadre réglementaire et sur l'absence d'une politique à l'échelle du massif, ainsi que sur l'importance d'une approche pluridisciplinaire des enjeux environnementaux.

\footnotetext{
${ }^{1}$ Cette recherche a donné lieu à une communication au colloque "Les mondes ruraux à l'épreuve des sciences sociales » (Dijon, INRA, 17-19 mai 2006), sous le titre : «Sentiments de la nature et expertises techniques dans la gestion de l'environnement. L'exemple des décisions de brûlage dirigé dans les Alpes méridionales françaises ".
}

\section{Le brûlage dirigé : une technique récente de gestion de l'environnement}

Le dépeuplement des régions de montagne à partir de la fin du XIX ${ }^{\mathrm{e}}$ siècle a entraîné l'abandon de grandes étendues de territoire autrefois exploitées dans un but agricole, pastoral ou forestier. Cette déprise agricole, phénomène généralisé aux montagnes de l'Europe occidentale (Cernusca et al., 1999), a conduit à une recolonisation par la végétation ligneuse des anciens parcours et des terres cultivées, qui se sont transformés progressivement en friches, en landes fermées ou en jeunes forêts selon l'ancienneté de l'abandon et les conditions écologiques locales. Cette fermeture des milieux, processus qui s'est accéléré dans la deuxième partie $d u X X^{\mathrm{e}}$ siècle, pose des problèmes écologiques et sociaux : risques accrus d'incendie à l'échelle du massif, perte de la biodiversité révélée par les scientifiques (Génot et Barbault, 2004), dégradation du paysage perçue par les résidents (Guisepelli et Fleury, 2003). Pour la plupart des scientifiques et des gestionnaires de l'environnement, aujourd'hui, la principale conséquence de la forte régression des milieux ouverts $\mathrm{d}^{\prime}$ altitude est le risque, si cette tendance à l'enfrichement ne s'infléchit pas, de voir décroître ou disparaître les es-

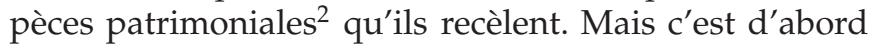
pour combattre les incendies, souvent dus à des feux pastoraux non maîtrisés, que s'est mise en place depuis les années quatre-vingt en France une politique de brûlage dirigé (Encadré 1).

Au cours du XXe siècle, le savoir-faire des populations rurales dans l'emploi du feu (Sigaut, 1975), toléré par le Code forestier dans le cadre des droits de propriété, mais longtemps combattu par l'administration des Eaux et Forêts (Ribet, 2005), a fortement régressé, en même temps que le feu pastoral se transformait de pratique collective en acte individuel le plus souvent dissimulé (Duché et Rigolot, 2004). Récemment, le feu a fait l'objet d'une requalification comme technique de gestion de l'environnement (Ribet, 1999), pour répondre à des objectifs variés : pastoraux en majorité ${ }^{3}$, mais aussi cynégétiques, touristiques, sylvicoles et environnementaux (Lambert, 1999). En effet, le feu est devenu non seulement un outil d'entretien des milieux pastoraux, mais aussi un " outil au service de la biodiversité » (Ribet, 2005), un outil polyvalent destiné à maintenir ou à restaurer des paysages au sens large, dans une optique qui allie gestion économique du

\footnotetext{
${ }^{2}$ Une espèce «patrimoniale » est une espèce qui bénéficie d'un statut réglementaire d'espèce menacée et/ou d'espèce protégée, ou encore qui est déclarée espèce rare par des experts.

3 Si la loi ne mentionne que la résorption des causes des incendies pour légitimer la pratique du brûlage dirigé, il faut remarquer que la très grande majorité des brûlages dirigés effectués dans la vingtaine de départements où il est pratiqué répondent à une demande formulée par un éleveur ou un berger, après accord du propriétaire du terrain.
} 
Tableau 1. Récapitulatif des personnes enquêtées.

\begin{tabular}{|c|c|c|}
\hline Protagonistes & Dates des entretiens & $\begin{array}{l}\text { Nombre et localisation des entretiens } \\
\text { (vallée, département) }\end{array}$ \\
\hline Éleveurs & De juin 2003 à mai 2005 & $\begin{array}{l}12 \text { entretiens (dont } 3 \text { répétés) dans } \\
4 \text { vallées du } 06 \text { (Vésubie, Cians, } \\
\text { Roya, moyen Var) et } 1 \text { entretien } \\
\text { dans le } 04\end{array}$ \\
\hline Pastoralistes & Juin 2003, juillet 2004 & 2 entretiens, 1 pour le 06,1 pour le 04 \\
\hline Chasseurs & $\begin{array}{l}\text { De juin } 2003 \text { à } \\
\text { août } 2006\end{array}$ & $\begin{array}{l}9 \text { entretiens (dont } 2 \text { répétés) dans } \\
4 \text { vallées du } 06 \text { (Cians, Roya, haut } \\
\text { Var, moyen Var) }\end{array}$ \\
\hline $\begin{array}{l}\text { Unité spécialisée } \\
\text { DFCI de l'ONF } 06\end{array}$ & $\begin{array}{l}\text { De juillet } 2003 \text { à } \\
\text { août } 2006\end{array}$ & $\begin{array}{l}10 \text { entretiens (dont } 2 \text { répétés) au } \\
\text { siège de la DFCI et dans } 4 \text { vallées } \\
\text { du } 06 \text { (Roya, Vésubie, moyen Var, } \\
\text { haut Var) }\end{array}$ \\
\hline Sapeurs-pompiers & Juillet 2004, avril 2005 & $\begin{array}{l}2 \text { entretiens, } 1 \text { pour le } 06 \text { (haut } \\
\text { Var), } 1 \text { pour le } 04\end{array}$ \\
\hline $\begin{array}{l}\text { ONF - unités } \\
\text { territoriales } 06 \text { et } 04\end{array}$ & Juillet et octobre 2004 & $\begin{array}{l}2 \text { entretiens, } 1 \text { pour le } 04,1 \text { pour le } \\
06 \text { (Estéron et moyen Var) }\end{array}$ \\
\hline $\begin{array}{l}\text { Maires de petites } \\
\text { communes }\end{array}$ & $\begin{array}{l}\text { De mai } 2004 \text { à } \\
\text { octobre } 2005\end{array}$ & $\begin{array}{l}3 \text { entretiens, } 2 \text { pour le } 06 \text { (Cians et } \\
\text { haut Var), } 1 \text { pour le } 04\end{array}$ \\
\hline $\begin{array}{l}\text { Parc national du } \\
\text { Mercantour }\end{array}$ & De mai à juillet 2004 & $\begin{array}{l}4 \text { entretiens, } 3 \text { dans le } 06 \text { (Vésubie, } \\
\text { Roya), } 1 \text { dans le } 04 \text { (Ubaye) }\end{array}$ \\
\hline $\begin{array}{l}\text { Associations de protection } \\
\text { de la nature }\end{array}$ & Avril et mai 2005 & 2 entretiens, 1 dans le 04,1 dans le 06 \\
\hline $\begin{array}{l}\text { Conseil général } 06 \\
\text { (direction de l’Écologie } \\
\text { et du Développement } \\
\text { durable) }\end{array}$ & Juin 2003, juin 2004 & 2 entretiens (06) \\
\hline Propriétaires & Février et mars 2005 & $\begin{array}{l}2 \text { entretiens dans le } 06 \text { (moyen Var, } \\
\text { Roya) }\end{array}$ \\
\hline
\end{tabular}

Les 50 entretiens ont été réalisés par 5 enquêteurs : outre Ariane Bernard-Laurent et Florence Weber (qui ont mené 15 entretiens ensemble, 8 ayant été conduits par A. B.-L. seule), il s'agit de Matthieu Marquet (9 entretiens), Aurélien Prudor et Aurore Seignemartin (16 entretiens ensemble, 2 par A. S. seule).

N. B. : huit personnes ont été interviewées à plusieurs reprises, parfois par des enquêteurs différents.

territoire et conservation du patrimoine naturel. Les travaux des scientifiques sur l'impact du feu (végétation, sol, faune) dans les écosystèmes méditerranéens ont contribué à cette "réhabilitation écologique » du feu : ils ont en effet montré qu'il pouvait être à l'origine d'un accroissement de la diversité floristique et faunistique (pour une revue de littérature, $c f$. Botelho, 2000). Dans le même temps, il cessait d'être un droit exclusivement dérivé du droit de propriété pour devenir une pratique légitimée par la puissance publique au titre de l'entretien de l'environnement. Dans ce basculement du rapport de force entre le propriétaire et l'État (qui garantit la professionnalisation de la pratique), les échelons intermédiaires, communes d'Ancien Régime, massifs géographiques ou instances régionales, restent remarquablement discrets.

Le brûlage dirigé est notamment mis en œuvre pour restaurer ou maintenir des habitats favorables à la faune sauvage ${ }^{4}$. Comme toute intervention sur le milieu, le brûlage a des effets directs et indirects et son bon usage à des fins de gestion de la faune et de ses habitats nécessite d'en connaître les conséquences, afin d'en affiner les modes opératoires et les prescriptions en fonction des espèces et des milieux. C'est précisément pour répondre à cet objectif que l'ONCFS a lancé, en 2002, une étude sur les incidences du feu sur l'habitat de la perdrix bartavelle, Alectoris graeca saxatilis, espèce patrimoniale.

Cette étude biologique a été le point de départ de notre enquête ethnographique (Encadré 2 ), car, au travers des relations établies avec les professionnels du brûlage

\footnotetext{
${ }^{4}$ Citons, à titre d'exemples, les brûlages réalisés pour améliorer l'habitat de diverses espèces d'oiseaux, tels la perdrix grise des Pyrénées (Novoa et al., 1998), la perdrix bartavelle dans les Alpes, l'aigle de Bonelli dans le Lubéron, le butor étoilé en Camargue.
} 


\section{Encadré 1. Qu'est-ce que le brûlage dirigé?}

Le brûlage dirigé est une technique de débroussaillement à faible coût, qui vise à résorber le nombre et l'importance des incendies. Il s'agit de l'utilisation d'un feu courant et maîtrisé dans le but de brûler la couverture végétale de manière plus ou moins intensive en fonction des conditions météorologiques, du type de végétation et des objectifs d'impact fixés. Le brûlage dirigé a été reconnu en tant qu'outil de prévention des incendies de forêt dans la dernière loi d'orientation sur la forêt (Loi n 2001-602 du 9 juillet 2001). Le décret $n^{\circ}$ 2002-679 du 29 avril 2002 indique que le brûlage dirigé désigne « la destruction par le feu des herbes, broussailles, litières, rémanents de coupe, branchages, bois morts, sujets d'essence forestière ou autres, lorsqu'ils présentent de façon durable un caractère dominé et dépérissant, dont le maintien est de nature à favoriser la propagation des incendies »; il précise que " cette opération est conduite de façon planifiée et contrôlée sur un périmètre prédéfini, avec obligation de mise en sécurité vis-à-vis des personnes, des biens, des peuplements forestiers et des terrains limitrophes, conformément aux dispositions du cahier des charges approuvé par chaque préfet de département ».

La période d'autorisation de brûlage, identique pour les opérations de feu contrôlé et pour celles conduites à titre privé, est fixée dans chaque département par arrêté préfectoral. Le brûlage dirigé est pratiqué le plus souvent de novembre à mars.

On peut distinguer trois modalités de la pratique de ce type de brûlage, qui témoignent de l'ambiguïté de sa définition, entre mission de service public et droit de propriété :

- Dans certains départements (dont les Alpes-Maritimes et les Alpes-de-Haute-Provence), le brûlage dirigé est effectué par des équipes professionnelles de composition variée (forestiers-sapeurs, sapeurs-pompiers, forestiers de l'ONF ou de la DDAF, militaires, éleveurs, gestionnaires de milieux naturels...), encadrées par une personne ayant reçu une formation spécialisée de "responsable de chantier de brûlage dirigé». Ces équipes, présentes aujourd'hui dans une vingtaine de départements, échangent leurs expériences au sein d'un «réseau national des équipes de brûlage dirigé », créé en 1990 puis animé jusqu'en 2005 par l'Unité de recherches forestières méditerranéennes de l'INRA d'Avignon (Rigolot, 1998). Récemment, le SIME (Syndicat interdépartemental montagne-élevage) Languedoc-Roussillon a pris le relais de la coordination de ce réseau.

- Dans d'autres départements, la mise en œuvre du brûlage dirigé est exécutée par un ou plusieurs particuliers, généralement des éleveurs (cas des chantiers à faible risque dans les Alpes-de-Haute-Provence, par exemple), sous le contrôle des professionnels de la sécurité. Dans ces deux premiers cas, l'accord du propriétaire est nécessaire. C'est lui qui est responsable en cas de débordement sur des parcelles voisines.

- Ailleurs, le droit de brûlage restant inscrit dans le Code forestier au titre des droits de propriété, tout propriétaire ou son ayant-droit peut effectuer des opérations de brûlage sous sa responsabilité et après déclaration en mairie. C'est la forme la plus répandue de brûlage dans les départements pastoraux pyrénéens (Métailié et Faerber, 2003). Il est très peu pratiqué dans les Alpes-de-Haute-Provence, car la cellule «brûlages dirigés » est en mesure de prendre en charge la totalité des demandes de brûlage. Dans les Alpes-Maritimes, les feux pastoraux hors cadre institutionnel sont si rares que leur légalité même constitue une surprise pour certains professionnels du brûlage dirigé enquêtés.

Nous n'étudierons ici que les deux premiers cas, qui représentent deux modalités d'institutionnalisation de la pratique : professionnalisation ou contrôle.

dirigé (tant praticiens que décideurs), mais aussi avec des usagers et des visiteurs des territoires brûlés ou à brûler, il nous est vite apparu que l'utilité, voire la légitimité de cette "pratique institutionnelle du feu » (selon l'expression de Ribet, 2005) était souvent contestée à l'échelle locale : les débroussaillements par le feu sont perçus par les citadins et les protecteurs de la nature comme une atteinte au paysage, à la faune et à la flore, tandis que d'autres acteurs, notamment éleveurs et bergers, les jugent insuffisants au regard de leurs besoins. La gestion des feux s'accompagne de conflits sur le «bon » usage des espaces d'altitude : c'est le cas, par exemple, dans les Pyrénées où des opérations de concertation ont été engagées dans chaque département pour remédier aux situations de blocage, en cherchant à améliorer les pratiques et à concilier l'usage du feu avec les divers enjeux locaux non pastoraux (Métailié et Faerber, 2003).

\section{L'organisation du feu contrôlé dans le Mercantour}

Le massif du Mercantour, adossé à la frontière du Piémont italien, est un espace montagneux tout proche de la Méditerranée, qui abrite une flore, une faune et des paysages d'une grande variété, ce qui a contribué à la création du Parc national du Mercantour (PNM) en 1979 sur une partie de son étendue. Le Mercantour s'étend sur deux départements limitrophes, les Alpes-Maritimes et les Alpes-de-Haute-Provence, dans lesquels l'organisation du feu contrôlé diffère radicalement. Le tropisme côtier des Alpes-Maritimes d'un côté, l'importance des enjeux agricoles dans les Alpes-de-Haute-Provence de l'autre, expliquent à la fois les différences entre les pratiques des éleveurs dans les deux départements et la présence de professionnels spécifiques du brûlage dirigé dans les Alpes-Maritimes (Tab. 2).

\section{Deux départements institutionnellement différents}

Dans les Alpes-Maritimes, les activités de brûlage ont été mises en place à partir de 1989 par un service de $1^{\prime} \mathrm{ONF}$ dénommé $\mathrm{DFCI}^{5}$ (Défense des forêts contre

\footnotetext{
5 Appellation identique à celle désignant la politique publique de défense des forêts contre l'incendie mise en place dans les années 1970.
} 


\section{Encadré 2. Enquête de terrain et coopération interdisciplinaire}

En 2003 et 2004, trois étudiants en gestion de l'environnement ont réalisé, sous la direction d'Ariane Bernard-Laurent, chercheur en biologie des populations, et parallèlement à l'étude technique qu'elle effectuait à l'ONCFS, 27 entretiens menés à l'aide d'une grille d'entretien auprès de personnes impliquées dans le processus de brûlage dirigé ou concernées par sa réalisation, dans la partie Alpes-Maritimes du massif du Mercantour. Issus pour la plupart du réseau d'interconnaissance professionnelle tissé par la biologiste, les enquêtés ont profité de l'enquête pour exprimer leurs doutes ou leurs désaccords sur les politiques et les pratiques du brûlage dirigé, ou pour renforcer ce qu'ils percevaient comme une sorte de légitimation scientifique de leurs positions. Dans un second temps, en 2004 et 2005, les deux auteurs de cet article ont réalisé ensemble 15 entretiens enregistrés, à la fois dans les Alpes-Maritimes et dans les Alpes-de-Haute-Provence, ce qui a permis de comparer les enjeux et le fonctionnement institutionnel du brûlage dirigé dans ces deux départements ( $c f$. Tab. 2). Enfin, au cours de l'été 2005, nous avons assisté à plusieurs réunions de concertation organisées par le conseil général, pour résoudre ce que nous appellerons des conflits de brûlage dirigé.

Ce dispositif d'enquête présente deux traits originaux. Il repose sur une coopération étroite entre une sociologue non impliquée et une biologiste, elle-même appelée à donner un avis technique sur le programme prévisionnel des brûlages dans le département. Le déroulement de l'enquête s'est accompagné d'une transformation des pratiques observées : après un exposé à mi-parcours de ses résultats préliminaires, plusieurs réunions de concertation ont été organisées pour la première fois en 2005.

l'incendie), dans une logique de « résorption des causes d'incendies ». Ces activités visaient explicitement à substituer aux éleveurs des équipes spécialisées, afin d'éviter les feux non maîtrisés aux conséquences lourdes en termes de vies humaines, de coût économique et d'atteinte à l'environnement. Pour la réalisation des chantiers de brûlage, ce service s'appuie sur un corps de forestierssapeurs, FORCE $06^{6}$ (Force opérationnelle risques, catastrophes, environnement des Alpes-Maritimes), composé de 186 agents en 2006, encadrés par l'ONF, et dont le financement est assuré par le conseil général.

C'est la DFCI qui reçoit les demandes effectuées par un maire, ou par une société de chasse, mais plus souvent par un éleveur. Ces demandes passent par plusieurs filtres : la DFCI elle-même évalue la faisabilité du chantier; elle sollicite l'accord des propriétaires des parcelles concernées (dans le cadre du droit de propriété dont relève toujours l'usage du feu), l'accord du maire (qui représente un échelon incontournable de la puissance publique) et, depuis peu, l'avis des experts (CERPAM [Centre d'études et de réalisations pastorales Alpes-Méditerranée], ONCFS, ainsi que le PNM si la demande est en zone centrale du Parc). Ce dispositif a bien fonctionné : les feux de landes et de friches qui dégénéraient en incendies de grande ampleur, nécessitant des interventions coûteuses des pompiers au cours des années 1980, sont devenus rarissimes.

Dans les Alpes-de-Haute-Provence, depuis 1993, la cellule «brûlages dirigés » poursuit, comme la DFCI 06, l'objectif d'ouverture d'espaces dans un but d'entretien des pâturages et de prévention des incendies, mais elle s'attache également à sensibiliser les éleveurs aux

\footnotetext{
${ }^{6}$ Le service FORCE 06 a été rattaché au département des Alpes-Maritimes le $1^{\text {er }}$ janvier 2007, mais demeure sous la tutelle technique de l'ONF. Il réalise des travaux de débroussaillement et des chantiers de brûlage dirigé en hiver, entretient les équipements de lutte contre les incendies, participe à la surveillance des forêts en été, intervient dans l'extinction des feux dans des endroits difficilement accessibles et aussi lors d'opérations exceptionnelles (inondations, pollutions...).
}

pratiques du brûlage en termes de technique, de sécurité et d'écologie, afin de les responsabiliser et de leur permettre d'effectuer eux-mêmes les mises à feu dans des conditions optimales d'efficacité et de sécurité et dans le respect de la réglementation. Aujourd'hui, les responsables de la cellule estiment que cet aspect pédagogique a porté ses fruits : les demandes de brûlage des éleveurs en termes de surface et de périodicité sont plus rationnelles, et bon nombre d'entre eux savent comment tirer le meilleur parti de la parcelle traitée pour assurer les besoins de leurs troupeaux.

La cellule fonctionne sur la base d'un recensement annuel des demandes auprès de tous les éleveurs des trois régions agricoles du département les plus sensibles aux risques d'incendies. Trace de l'importance des activités pastorales dans l'économie des Alpes-de-HauteProvence, ce sont les organismes à vocation agricole (la DDAF 04 [direction départementale de l'Agriculture et de la Forêt] et le CERPAM 04, organisme de développement pastoral) qui ont le rôle décisionnel majeur dans la planification des opérations de brûlage, aux côtés des sapeurs-pompiers. Au contraire, la DDAF 06 n'est même pas consultée sur l'opportunité des demandes de brûlage, ce qui révèle le très faible poids économique et social du pastoralisme dans les Alpes-Maritimes (Legeard et Mallen, 1996).

La gestion administrative des activités de brûlage apparaît plus simple dans les Alpes-de-Haute-Provence que dans les Alpes-Maritimes pour au moins deux raisons : c'est la DFCI 06 qui se charge de rechercher les propriétaires des parcelles à brûler et de les solliciter par écrit pour obtenir leur autorisation de brûler, alors que dans les Alpes-de-Haute-Provence, le dossier de demande de brûlage que l'éleveur transmet à la cellule doit être accompagné d'une autorisation du propriétaire ; par ailleurs, les enjeux écologiques au sens large sont pris en compte de façon systématique depuis 2004 dans le dispositif des Alpes-Maritimes, ce qui n'est pas le cas dans les Alpes-de-Haute-Provence. 
Tableau 2. Dispositifs administratifs du brûlage dirigé dans les Alpes-Maritimes et les Alpes-de-Haute-Provence.

\begin{tabular}{|c|c|c|}
\hline & $\begin{array}{l}\text { Atelier départemental } \\
\text { de brûlage dirigé des } \\
\text { Alpes-Maritimes }\end{array}$ & $\begin{array}{l}\text { Cellule «brûlages dirigés » des } \\
\text { Alpes-de-Haute-Provence }\end{array}$ \\
\hline $\begin{array}{l}\text { Composition de la cellule instruisant } \\
\text { les demandes }\end{array}$ & $\begin{array}{l}\text { CG } 06 \text { maître d'ouvrage, } \\
\text { DFCI maître d'œuvre (conven- } \\
\text { tion département-ONF) }\end{array}$ & $\begin{array}{l}\text { DDAF 04, SDIS, ONF 04, } \\
\text { CERPAM } 04\end{array}$ \\
\hline Recensement des demandes & DFCI & Demandeurs \\
\hline $\begin{array}{l}\text { Recueil des autorisations de } \\
\text { propriétaires }\end{array}$ & DFCI & CERPAM 04 \\
\hline Commission de concertation & $\begin{array}{l}\text { CG 06, DFCI, CERPAM 06, } \\
\text { PNM, ONCFS }\end{array}$ & $\begin{array}{l}\text { DDAF 04, SDIS, ONF 04, } \\
\text { CERPAM } 04\end{array}$ \\
\hline Personnel exécutant les feux contrôlés & $\begin{array}{l}\text { Forestiers-sapeurs (FORCE 06), } \\
\text { encadrés par l'ONF }\end{array}$ & $\begin{array}{l}\text { Éleveurs / SDIS USC 7/APFM } \\
\text { selon le niveau de risque du } \\
\text { chantier }\end{array}$ \\
\hline Financement des opérations & $\begin{array}{l}\text { Département (90 \%), CFM État } \\
(10 \%)\end{array}$ & $\begin{array}{l}\text { Département (79 \%), CFM État } \\
(21 \%)\end{array}$ \\
\hline $\begin{array}{l}\text { Bilan quantitatif pour la période } \\
2000-2004 \text { : } \\
\text { - nombre moyen annuel de chantiers } \\
\text { - nombre moyen de communes } \\
\text { - surface moyenne traitée annuellement }\end{array}$ & $\begin{array}{l}50 \\
34 \\
1582 \text { ha }\end{array}$ & $\begin{array}{l}23 \\
13 \\
241 \text { ha }\end{array}$ \\
\hline Suivis réalisés après brûlage dirigé & $\begin{array}{l}\text { Suivi des surfaces pastorales } \\
\text { brûlées dans la vallée de la Roya } \\
\text { (convention département- } \\
\text { CERPAM 06) et compte rendu } \\
\text { technique annuel }\end{array}$ & $\begin{array}{l}\text { Suivi pastoral par le } \\
\text { CERPAM } 04 \text { de } 1 \text { ou } 2 \text { parcelles } \\
\text { traitées par brûlage chaque année } \\
\text { et compte rendu technique annuel }\end{array}$ \\
\hline
\end{tabular}

Lecture des acronymes :

APFM : Auxiliaires de protection de la forêt méditerranéenne (sous la responsabilité de l'ONF)

CERPAM : Centre d'études et de réalisations pastorales Alpes-Méditerranée

CFM : Conservatoire de la forêt méditerranéenne

CG : conseil général

DDAF : direction départementale de l'Agriculture et de la Forêt

DFCI : Défense des forêts contre l'incendie (unité spécialisée de l'ONF 06)

FORCE 06 : Force opérationnelle risques, catastrophes, environnement des Alpes-Maritimes

ONCFS : Office national de la chasse et de la faune sauvage

ONF : Office national des forêts

PNM : Parc national du Mercantour

SDIS : service départemental d'Incendie et de Secours

USC 7 : unité de sécurité civile basée à Brignoles, dans le département du Var

Pour la réalisation des chantiers, dans les Alpes-deHaute-Provence, il est fait appel aux sapeurs-pompiers locaux, aux militaires de l'unité de la sécurité civile du Var et aux auxiliaires de protection de la forêt méditerranéenne. Le personnel exécutant le brûlage dépend du niveau de risque du chantier : si les éleveurs sont propriétaires du terrain et si le risque du chantier a été jugé faible par un binôme constitué d'un agent ONF et d'un pompier, ils sont autorisés à réaliser seuls le brûlage et le font régulièrement.

Aucun autre département français que les AlpesMaritimes ne dispose de moyens aussi importants en personnel engagé dans des programmes de défense contre l'incendie. C'est probablement le résultat du fort dualisme du département entre la Côte d'Azur, véritable métropole côtière où sont concentrés tous les problèmes mais aussi toutes les ressources économiques et politiques liés à sa densité urbaine exceptionnelle (882 habitants $/ \mathrm{km}^{2}$ sur $1113 \mathrm{~km}^{2}$ ), et son arrière-pays (renommé Haut-Pays dans les années 1990) qui, avec une densité de 9,3 habitants $/ \mathrm{km}^{2}$ sur $3186 \mathrm{~km}^{2}$, constitue une sorte de «réserve» au sens colonial du terme. Dans ce département, la montagne est l'objet d'un attachement sentimental de la part des "enfants du pays » partis sur la bande côtière, mais restant résidents intermittents; elle est l'objet d'une sollicitude clientéliste de la part des hommes politiques locaux - parfois eux aussi des résidents intermittents. Les pouvoirs publics, élus et fonctionnaires confondus, souhaitent donc maintenir quelques ménages sur place, éleveurs ou employés 
des administrations publiques (dont ceux du service FORCE 06), pour garantir les conditions minimales d'une occupation saisonnière de l'espace par des citadins. En revanche, ils ne se soucient pas d'aider les résidents du Haut-Pays à se dégager de leur tutelle, comme en témoignent l'absence de politique de soutien à l'innovation ou aux entreprises, et même le manque de structures sanitaires, économiques et sociales minimales.

L'existence, dans les Alpes-Maritimes, d'une main d'œuvre importante, présente dans toutes les vallées du département, a plusieurs conséquences : la superficie annuelle brûlée est sept fois plus grande que dans les Alpes-de-Haute-Provence, pour seulement deux fois plus de chantiers (Tab. 2); et, surtout, les équipes de forestiers-sapeurs se sont presque totalement substituées aux éleveurs pour réaliser les opérations de brûlage. En effet, même si un arrêté préfectoral prévoit la possibilité pour un propriétaire ou son ayant-droit de réaliser lui-même un feu, sous réserve d'en obtenir l'autorisation par la mairie (et après consultation du chef de corps des sapeurs-pompiers et d'un agent de l'ONF), nous avons constaté que la plupart des éleveurs, n'étant pas propriétaires et disposant rarement de baux écrits pour leurs parcours, ignorent jusqu'à l'existence de cette procédure. De plus, la plupart d'entre eux ne possèdent pas le savoirfaire du feu contrôlé. C'est pourquoi ils apprécient l'existence d'un service de forestiers-sapeurs, qui leur permet d'agrandir ou d'améliorer leurs pâturages gratuitement, comme le montre le large réseau d'éleveurs constitué par le forestier-sapeur chargé de répertorier et de mettre en forme avant toute décision officielle les demandes, dont le nombre a fortement crû au fil du temps.

\section{Le brûlage dirigé, un métier?}

$\mathrm{Si}$, dans les Alpes-de-Haute-Provence comme dans d'autres départements - Hautes-Pyrénées (Métailié et Faerber, 2003) ou Lozère (Dumez, 2004), notamment -, le brûlage dirigé est une compétence directement transmise au sein du milieu pastoral, il n'en va pas de même dans les Alpes-Maritimes. Ici, bien peu d'éleveurs pratiquent le feu pastoral et, lorsqu'ils le font, ce n'est pas par souci d'affirmation de leur indépendance, mais bien plutôt par lassitude devant la lourdeur de la procédure et l'absence de disponibilité des forestiers-sapeurs. C'est ce qu'explique un vacher d'une vallée proche de l'Italie, qui a fini par effectuer ses brûlages seul avec sa femme, ses demandes ayant été refusées parce qu'elles «n'étaient pas dans les temps ». Il s'est donc débrouillé, retrouvant la pratique de l'écobuage utilisée par son père, employé communal, dans ses vergers.

« - Et la technique de brûlage, vous l'avez apprise de votre père?

- Par mon père et puis en voyant...
- Et vous choisissez des jours?

- Calmes si possible, sans vent, s'il y a la neige c'est encore mieux, quand il y a la neige on fait d'abord les talus et la fois d'après on fait les plats.

- Et vous êtes satisfait des résultats du brûlage pour les bêtes?

- Oui, c'est pas mal, si on pâture bien c'est définitif... Il faut surtout une bonne année pluvieuse, sûr que si c'est une année sèche, derrière c'est moins bon, ça on n'y peut rien.

- Et vous n'avez pas peur quand vous faites le brûlage vous-même?

- (Petit rire.) Quelquefois... On a toujours des frayeurs, mais bon, de toute manière ils peuvent pas aller de partout alors, la DFCI. Bon, on commence toujours par le haut... On fait de petits pare-feux, et puis s'il faut, on retourne 3-4 fois, on fait pas tout d'un coup, il vaut mieux y aller doucement... »

D'autres bergers transforment ce constat fataliste les forestiers-sapeurs ne sont pas disponibles, leurs machines sont en panne, les pompiers n'ont pas le droit de donner un coup de main - en une double critique : ils sont dépossédés de leur droit de brûler, alors qu'ils en auraient la compétence, au profit d'un service dont les employés déploient des précautions qui leur semblent disproportionnées.

De fait, les forestiers-sapeurs qui participent aux chantiers de brûlage entretiennent un rapport ambivalent avec le feu : d'un côté, ils aiment beaucoup leur métier ${ }^{7}$; de l'autre, ils sont conscients des risques qu'ils courent et racontent les accidents de brûlage, dont certains furent dramatiques; ils sont également conscients des critiques dont fait l'objet le brûlage dirigé et les reprennent à leur propre compte.

Dans les Alpes-Maritimes, du fait de la centralisation des demandes par la DFCI, une seule contestation, d'où qu'elle vienne, suffit en règle générale à bloquer le chantier. Cependant, nous avons pu observer un cas exceptionnel à plusieurs titres, puisqu'une contestation reçue par courrier, et relayée par certains experts de l'environnement, a suscité une réunion de concertation, véritable innovation institutionnelle dans le département, en partie liée au déroulement de notre enquête. Son observation nous a permis de mieux comprendre le processus de décision à l'œuvre dans les Alpes-Maritimes, dont la spécificité renvoie à un état particulier des rapports de

\footnotetext{
7 Tous les forestiers-sapeurs rencontrés nous ont renvoyé une image très positive de leur métier. Parmi bien d'autres citations possibles : «Le travail qu'on fait, moi, vraiment, c'est un travail qui est parfait, c'est vraiment ce que je cherchais, quoi ; on est en permanence dans la nature, on a un secteur qui est assez grand, ça nous permet de voir pas mal de pays, on est vraiment entre collègues de la vallée; au point de vue du travail, pour moi je suis vraiment content d'avoir ça. »
} 
force et de la division du travail entre éleveurs, élus et administrations. Malgré cette spécificité, ou peut-être à cause d'elle, il nous semble pouvoir mettre en évidence un résultat de portée générale : une décision de gestion de l'environnement, quelle qu'elle soit, suppose la combinaison de deux types de savoirs distincts, tous deux inégalement distribués, des savoirs pratiques liés à l'observation et à l'usage d'un territoire, des savoirs relationnels liés à la compréhension des enjeux politiques et sociaux.

\section{Un conflit autour du brûlage dirigé et sa résolution}

En 2004, le conseil général reçoit deux lettres de chasseurs opposés à la demande de brûlage effectuée par le maire de la commune de Vareddes $^{8}$, qui visait à la réouverture du milieu sur une colline isolée au confluent de deux vallées, arguant de l'intérêt du site pour l'éleveurberger local. L'argument majeur des opposants au brûlage de cette colline est le risque de destruction par le feu des genévriers cades, dont les fruits attirent les grives lors des migrations d'automne et qui font de ce site un lieu traditionnel de chasse à la grive pour les anciens du pays. Le conseil général transmet ces lettres au maire, accompagnées d'un avis de l'ingénieur pastoraliste peu favorable à la demande contestée. Deux mois plus tard, le maire réplique sur un ton courroucé, mettant en cause tant «l'égoïsme primaire » des contestataires que l'avis «frileux » du pastoraliste. Soucieux de ménager la susceptibilité de l'élu sans toutefois désavouer l'expert avec lequel il travaille, le chef du service concerné au conseil général propose alors une réunion de concertation, qui sera finalement organisée par la mairie de Vareddes au cours du printemps suivant.

\section{Chasse à la grive, intérêt pastoral et légitimité politique}

Comme d'autres communes de l'arrière-pays niçois, Vareddes est depuis longtemps un village de résidents intermittents : des personnes originaires du lieu, propriétaires de maisons et de terrains, habitent et travaillent sur la côte tout en étant fortement attachés au village, où ils reviennent dès qu'ils le peuvent. Difficile d'accès, Vareddes compte moins de 25 résidents permanents. Un éleveur-berger d'origine locale et sa femme sont installés sur le territoire communal et leur activité est fortement

\footnotetext{
${ }^{8}$ Les noms propres, de personnes et de lieux, ont été changés ainsi que plusieurs détails qui auraient permis l'identification des protagonistes des histoires racontées ici, qui ne valent pas pour elles-mêmes mais pour ce qu'elles révèlent des rapports de force locaux, d'une part, et du processus de décision ordinaire en matière de brûlage dirigé, d'autre part.
}

soutenue par la municipalité. Gestion de la commune et gestion de la société de chasse communale sont très imbriquées. La chasse connaît cependant une crise de reproduction. Majoritaires, les chasseurs de sangliers, des quinquagénaires, sont inquiets pour l'avenir : alors qu'ils poursuivent une politique de cultures à sangliers, l'un d'entre eux déplore qu'il n'y ait plus de jeunes chasseurs et explique que « bientôt il n'y aura plus de chasseurs et [qu']il faudra payer des gens pour tuer le gibier proliférant ». Minoritaires, les chasseurs de grives, plus âgés, ont trouvé un successeur, qui les a aidés à protester contre la demande municipale de brûlage visant l'un des meilleurs territoires de chasse à la grive. Ce jeune informaticien, Antoine Robbe, lui aussi résident intermittent, inscrit sa pratique de la chasse dans la durée :

«Moi j'ai un petit garçon, quand je l'ai amené làbas, il a six ans, bon voilà, moi j'ai appris à chasser grâce à la grive, je trouve qu'en plus la grive c'est un oiseau qui est beau, il y a les traditions dans la Provence autour de la chasse à la grive, il y a plein de choses, ça serait trop long si je commence à parler làdessus... C'est une chasse qui peut permettre un trait d'union entre toutes les générations, on commence à amener un enfant à la chasse à la grive vers 10-12 ans quand il veut commencer à aller au poste, et on finit à 75 ans quand on a du mal à marcher, aller finalement tirer quelques grives le matin, voilà, bon. Et moi j'ai toujours vu les vieux comme ça. »

Première lecture du conflit autour du brûlage, donc : cette crise de reproduction, cette lutte pour l'avenir de la chasse explique la virulence des accusations réciproques. Les chasseurs de sangliers accusent les chasseurs de grives d'insoumission au collectif : «Ils ne peuvent pas faire partie d'une équipe ", " ce sont des braconniers ", nous dit-on. Antoine Robbe, lui, disqualifie radicalement la pratique des chasseurs de sangliers :

«Soit la chasse arrivera à perdurer au travers de la culture de la chasse, soit elle disparaîtra, parce que aller chasser en $4 \times 4$ sur des pistes, aller se poster à un endroit pour tuer de grosses bêtes qu'on a élevées au maïs, ça n'a aucun intérêt. C'est pas de la culture, c'est de l'assassinat! »

Si l'on écoute le maire, lui-même chasseur de sangliers, ce conflit entre chasseurs est secondaire. Ce qui est en jeu à ses yeux, ce n'est pas l'avenir de la chasse, mais l'avenir de la commune, qui passe d'abord par le pastoralisme. Nul ne conteste le soutien municipal à l'éleveur local. Après cinquante ans de déclin des activités productives et de diminution des résidents permanents, quelques indices laissent penser que la pente pourrait s'inverser. Dans les années à venir, plusieurs résidents intermittents, arrivés à l'âge de la retraite, envisagent de s'installer à demeure. L'éleveur et sa femme, bien 
que d'origine rurale, sont eux-mêmes d'anciens salariés urbains qui partagent le goût de ces résidents intermittents pour le «traditionnel » et le « local». La question est de savoir quelles parcelles brûler pour favoriser l'activité pastorale. Si le maire a choisi la colline à grives, c'est en référence au passé cultural de la commune : la colline était cultivée, des troupeaux de chèvres et de moutons la fréquentaient.

Une seconde dimension, familiale cette fois, du conflit de brûlage apparaît ici. Le terrain appartenait au grandpère $d^{\prime}$ Antoine Robbe, qui était éleveur et fut longtemps le maire du village ; et c'est son père, devenu salarié qualifié en ville, qui l'a vendu à l'ONF. On peut supposer que la demande de brûlage dirigé formulée par le maire sur une parcelle chargée d'histoire sociale est un prétexte pour régler des comptes strictement locaux. La décision de vente de ce terrain, perçue comme une trahison par rapport à l'appartenance locale, disqualifie politiquement la parole de la lignée familiale concernée. Le maire insiste sur le fait que les contestataires ne sont pas propriétaires : ils n'ont doublement rien à dire sur l'usage d'une parcelle qu'ils ont abandonnée. Légalement, le droit de feu est lié au droit de propriété. Symboliquement, la légitimité politique s'acquiert difficilement (les nouveaux propriétaires sont peu écoutés), mais elle se perd facilement (vendre, c'est trahir). En réponse, Antoine Robbe invoque lui aussi le passé : ces terrains étaient pâturés par des chèvres et non par des moutons, ils sont difficiles d'accès et présentent des risques pour le troupeau. Mais, parlant du passé, il usurpe une compétence proprement politique, portée d'habitude par les élus locaux. De plus, en s'adressant directement aux administrations et en cherchant à s'immiscer dans le circuit de la décision administrative, il s'affranchit du registre des relations interpersonnelles et transgresse l'ordre local tacite qui réserve à l'élu la relation avec l'administration. Comme il le dit après coup, «les gens sont venus [me voir] en bloc, ils n'ont pas apprécié que je passe par une voie détournée».

\section{Compétences pratiques, compétences sociales et résolution des conflits}

L'évaluation de l'intérêt pastoral et, plus largement, écologique d'une parcelle peut aussi être l'occasion d'un conflit de compétences entre certains élus et certains techniciens. Des divergences de perception du territoire entre personnels de l'administration, techniciens et élus locaux ont pu être observées par la sociologue à l'occasion de la réunion de concertation organisée à Vareddes pour répondre à la demande du maire. Postés sur un belvédère difficile d'accès, techniciens, élus et responsables administratifs ont échangé, lors de discussions en petits groupes, leurs points de vue sur l'opportunité d'une remise en valeur par le feu de la parcelle concernée. Les élus présents, originaires de la commune, lisent le paysage en fonction de son histoire : ils y cherchent, non sans nostalgie, les pratiques passées qui l'ont façonné et la trace de leurs aïeux, pour affirmer que ce qui a été est toujours possible. En face, les techniciens, malgré la diversité de leurs compétences, lisent tous le paysage de la même façon : la nature des sols, les caractéristiques actuelles de la végétation (qui autorisent des hypothèses sur sa dynamique ancienne et sur sa réaction au feu), les caractéristiques du relief, sont mises en commun pour débattre de l'avenir de la parcelle au cours d'une discussion entre pairs qui évaluent ses potentialités faunistiques (c'est le rôle de la biologiste), pastorales (c'est celui de l'ingénieur pastoraliste) et les risques liés au brûlage lui-même (c'est celui des techniciens du feu). Le berger et sa femme, premiers concernés, sont présents mais interviennent peu dans la discussion. Leurs pâturages sont éloignés de ce site qui pourrait les intéresser pour la période hivernale, à condition que sa superficie pâturable soit de taille suffisante.

Les arguments qui s'échangent alors entre techniciens portent sur la nature elle-même et non sur la légitimité sociale à en parler. L'expérience pratique - avoir parcouru ce terrain-là, l'avoir utilisé, l'avoir vu réagir au fil du temps - est alors tout aussi légitime que le savoir fondé sur des connaissances théoriques, lui-même reconnu seulement s'il autorise des conclusions appliquées hic et nunc. De ce fait, un berger, un résident, un chasseur, s'il dispose d'une expérience pratique, peut la voir reconnue et validée par les professionnels. C'est d'ailleurs ce qui a amené les experts, " off magnéto », à s'intéresser à la contestation rédigée par Antoine Robbe : son argumentaire sur le passage des grives était parfaitement recevable, et le fait qu'il était fondé sur l'ancienneté des observations et non sur une compétence scolairement certifiée ne pouvait en aucun cas gêner des professionnels dont le savoir scientifique - ornithologie et pastoralismerepose justement sur une alliance originale entre amateurs et professionnels, entre savoirs locaux et science légitime. Au contraire, les chasseurs de sangliers se sont disqualifiés, aux yeux de ces mêmes experts, par leur méconnaissance évidente des enjeux liés à la conservation d'un territoire très favorable aux grives et du système pastoral de l'éleveur, qui n'avait pas grand intérêt à tirer du défrichement de cette zone.

Entre eux, les praticiens, dont la compétence relève de sciences différentes, voire d'aucun corps constitué de connaissances scientifiques, reconnaissent mutuellement la validité de leurs savoirs pratiques. Pour insister sur le caractère spécifique des savoirs qu'ils mettent en œuvre, indépendamment de toute légitimation sociale ou institutionnelle, nous appellerons ces savoirs des «savoirs pratiques » et nous considérerons qu'il existe un espace commun de compétences reconnues et complémentaires entre les professionnels, qui se nomment eux-mêmes des "techniciens », et des "praticiens » amateurs, qui disposent de compétences techniques étendues, dont ils font 
usage dans leur vie quotidienne (professionnelle ou de loisir), sans en tirer ni statut ni rémunération.

Ainsi, les praticiens du feu, en dehors de toute considération de position professionnelle et de hiérarchie institutionnelle, savent anticiper les réactions du sol à l'action $\mathrm{du}$ feu; de ce point de vue, le clivage ne passe pas entre professionnels du feu et éleveurs, mais entre praticiens du feu expérimentés ou non, les savoirs techniques sur le feu étant fort inégalement distribués entre éleveurs, mais aussi entre forestiers-sapeurs, selon leur pratique de l'activité de brûlage. Les praticiens du pastoralisme, qu'ils soient ingénieurs ou techniciens salariés d'organismes agricoles ou encore simples éleveurs, savent anticiper les réactions du troupeau confronté à la parcelle. Les praticiens de la faune sauvage, qu'il s'agisse d'un ingénieur, d'agents d'exécution des organismes de gestion et de contrôle de la chasse, ou de certains chasseurs, savent anticiper la dynamique des populations de gibier dont ils sont bons connaisseurs.

Les différences sociales, et même les relations hiérarchiques, qui sont importantes en toute autre occasion, sont mises entre parenthèses le temps de cette discussion technique. L'estime mutuelle que se portent l'ingénieur pastoraliste et certains bergers, la biologiste et certains chasseurs, l'ingénieur forestier et certains forestiers-sapeurs, est circonscrite à l'espace de telles discussions. Pour autant, elle explique entièrement la capacité de chacun à écouter les autres, alors qu'ils se révèlent, sur d'autres scènes sociales, de farouches adversaires. Par exemple, l'acrimonie, sensible dans leurs relations institutionnelles, entre le chef des forestiers-sapeurs et l'agent subalterne chargé de préparer les dossiers de brûlage qui se trouve être également une figure du syndicalismedisparaît dans ce contexte spécifique. Ou encore, la sympathie ou les oppositions, liées à leurs proximités idéologiques et sociales ou à leurs divergences politiques et à leur distance de classe, qui existent par ailleurs entre certains techniciens (pastoraliste, biologiste) et certains praticiens amateurs (bergers, chasseurs), font place, sur la scène technique délimitée par un lieu, un moment et une action spécifiques, à une estime mutuelle où chacun ne vaut que pour l'argument qu'il est en train d'avancer.

Cette réunion de concertation fut donc l'occasion de réaffirmer les compétences techniques des professionnels de l'environnement, qu'il s'agisse de personnels des administrations directement concernées (conseil général, ONF) ou d'autres organismes consultés (CERPAM, ONCFS). Mais, avant même que la discussion technique ait lieu, débouchant sur un accord relatif à la bonne décision à prendre, la présence des responsables des deux administrations, ainsi que le caractère officiel de la rencontre, marqué par l'omniprésence des sigles de l'administration (sur les uniformes et sur les voitures), avaient suffi à montrer le soutien apporté par les institutions à la parole des techniciens.
Raison technicienne et soutien institutionnel ont permis de régler provisoirement ce conflit à deux dimensions. À l'échelle locale, il opposait un chasseur de grives, mis en accusation pour n'être pas resté propriétaire et avoir trahi la cause du pays, à un maire engagé dans le soutien actif à « son » berger. À l'échelle départementale, et de façon plus structurelle, il s'agissait d'un conflit de légitimité opposant élus et administration : légitimité politique de l'élu municipal et légitimité technicienne d'une administration dépendant elle-même d'une assemblée départementale élue, le conseil général. La complexité de ce deuxième conflit a été gérée tout en finesse par l'ensemble des personnes concernées : le maire ne devait surtout pas perdre la face, ni avoir recours aux élus départementaux. Les réserves concernant le terrain (trop éloigné du parcours de l'éleveur) ont conduit à doubler la surface à réouvrir. Les réserves concernant le feu (risques d'éboulement, difficulté à mettre hors feu les arbustes nourriciers des grives, difficultés d'accès en cas de débordement) ont conduit à adopter une solution beaucoup plus coûteuse, le débroussaillage mécanique. La décision finale restait suspendue à deux points techniques : il fallait l'accord du responsable du débroussaillage mécanique, qui fut obtenu; il fallait qu'il y ait un point d'eau, comme l'affirment ceux qui ont connu le terrain lorsqu'il était cultivé. Le service des forestiers-sapeurs souhaite en profiter pour transformer ce point d'eau en réservoir et s'en servir à l'échelle de la vallée pour les opérations de lutte contre l'incendie. À cette occasion, on découvre que le responsable du service a, sur le paysage, l'œil du militaire en campagne : il évalue immédiatement non pas tant les enjeux symboliques ni même les enjeux environnementaux que les enjeux pratiques liés au déploiement dans l'espace de ses troupes. En 2006, deux hectares ont été débroussaillés, avec la promesse de poursuivre, alors que la demande de brûlage portait sur une trentaine d'hectares. Le conflit s'est réglé à la satisfaction générale. Cependant, la surface est trop petite pour répondre aux besoins du berger, qui effectue par ailleurs des demandes de brûlage pour ses pâturages habituels.

\section{Conclusion}

Les différents enjeux du brûlage dirigé justifient son analyse par plusieurs disciplines : économie de l'agriculture, de l'espace rural et de l'environnement (emplois agricoles et environnementaux, production pastorale et ses externalités, terme qui désigne en économie la " production involontaire d'environnement $»$ ), sociologie du travail, des savoirs et de la politique (rapport des différents professionnels concernés à leur métier, savoirs pratiques, techniques et scientifiques, rapports de force entre élus, administrations et citoyens), écologie (effets sur le milieu). C'est pourquoi il suppose une approche véritablement interdisciplinaire, comme le souligne Barbault 
(2006) pour l'ensemble des objets de l'écologie. De ce point de vue, notre enquête expérimente une modalité particulière de l'interdisciplinarité : elle est fondée sur un respect mutuel pour nos compétences respectives, forgé dans la durée, et sur la complémentarité entre l'engagement local de la biologiste et la distance aux enjeux locaux de la sociologue (touriste dans la région). Qu'apporte-telle à la connaissance de la gestion de l'environnement ?

Conçu dans les Alpes-Maritimes comme un moyen de lutte contre les incendies et dans les Alpes-de-HauteProvence comme une aide à la profession agricole, le brûlage dirigé est aujourd'hui requalifié par une partie des professionnels de l'environnement comme un moyen d'entretien de la montagne.

Tous les éleveurs interrogés souhaitent un usage plus fréquent et moins contraignant du feu pour gagner gratuitement des surfaces pâturables, mais surtout pour lutter contre l'envahissement de leurs pâturages par les broussailles, la dent du bétail ne parvenant pas à elle seule à conserver les alpages dans un état "propre » (Dumez, 2004). Pour autant, certains se contenteraient volontiers d'en déléguer la tâche, ingrate et dangereuse, à des professionnels du feu. Dès lors, la question principale porte sur les coûts de la pratique : qui doit payer pour une activité destinée, principalement ou secondairement, à des fins pastorales? Comment «calculer » la multiplicité des conséquences bénéfiques d'une pratique qui a un coût, même lorsqu'il reste invisible? Pour prendre la décision politique de déterminer qui doit effectuer le travail de brûlage, et dans quelles conditions (obligation d'entretien du propriétaire, tâche annexe de l'éleveur, contre rémunération ou non, tâche principale du sapeur-forestier, rémunérée par l'impôt au titre de l'intérêt général), il n'est pas sans intérêt d'observer la division du travail du feu selon les contextes professionnels et administratifs et de s'interroger sur son bien-fondé.

Les professionnels de l'environnement restent partagés. Dans une période caractérisée par une transformation des idées sur la protection de la nature, différentes positions idéologiques mais aussi différents niveaux d'information, tant scientifique que politique, coexistent au sein d'une même structure ou entre institutions appelées à coopérer. Ainsi, au sein du Parc national du Mercantour, des divergences marquées d'appréciation sur le feu sont apparues entre les scientifiques, souvent proches du pouvoir de décision, plutôt favorables au brûlage dirigé, et les agents de terrain, pour qui le feu reste synonyme de dégradation de l'environnement et dont la position se rapproche de celle des protecteurs de la nature, de certains chasseurs et des spectateurs urbains.

Enquêter sur le brûlage dirigé aujourd'hui, c'est entendre une multiplicité d'accusations croisées, portant toutes sur l'incompétence supposée des différents "producteurs » de l'environnement, que leur activité, professionnelle ou non, soit directement consacrée à la gestion de l'environnement ou qu'elle ait des incidences importantes sur les milieux naturels, comme les activités pastorales ou la chasse. C'est aussi être amené à distinguer, pour mieux comprendre la diversité des regards portés sur les paysages, souvent incompatibles (Larrère et Larrère, 1997), différents types de savoirs : des savoirs pratiques portant sur la nature, distribués de façon inégale, dont les détenteurs disposent d'une reconnaissance mutuelle accordée par leurs pairs, sans considération de statut professionnel ou de certification scolaire ou administrative; mais aussi des compétences relationnelles, ou savoirs sociaux, eux aussi distribués de façon inégale selon d'autres types de clivages, liés cette fois à la connaissance fine des arcanes institutionnelles et des enjeux politiques locaux, au sens large du terme.

C'est enfin se poser la question de l'échelle pertinente de l'intervention publique. On ne peut qu'être frappé par l'inadéquation entre, d'une part, l'échelle communale à laquelle se prennent les décisions de brûlage dirigé, l'échelle départementale qui leur sert à la fois d'arène politique et de cadre réglementaire, et, d'autre part les enjeux écologiques et sociaux qui se jouent, eux, à l'échelle du massif. On est conduit à se demander si les pouvoirs publics ne pourraient pas proposer un «plan d'ouverture des paysages » à l'échelle du massif, c'est-à-dire à la fois pluricommunal et bidépartemental, qui donnerait une cohérence aux multiples demandes provenant de municipalités, d'éleveurs ou de chasseurs. Ce plan d'ouverture des paysages pourrait servir de cadre aux décisions de brûlage dirigé, négociées commune par commune avec les principaux producteurs de l'environnement, avec le souci de prendre en compte l'ensemble des enjeux économiques, territoriaux, sociaux et environnementaux induits par la déprise agricole. À condition, bien sûr, qu'un tel plan n'ajoute pas une couche supplémentaire à la complexité institutionnelle qui caractérise aujourd'hui la gestion de l'environnement, mais qu'il soit envisagé comme la seule échelle pertinente de décision portant sur le brûlage dirigé, voire - nul n'empêche de rêver sur l'environnement.

\section{Remerciements}

Nous remercions Matthieu Marquet, Aurélien Prudor et Aurore Seignemartin, qui ont effectué la première phase de l'enquête. Nous sommes reconnaissantes à l'ensemble des personnes qui ont accepté de participer à notre enquête, qui nous ont consacré du temps et nous ont fait confiance, et en particulier aux éleveurs du Haut-Pays niçois, aux membres de la DFCI 06, du conseil général et du CERPAM. Nous remercions les participants au colloque "Les mondes ruraux à l'épreuve des sciences sociales » (Dijon, INRA, 17-19 mai 2006), particulièrement Susan Carol Rogers, les participants à la séance du 19 décembre 2006 de l'atelier «Biodiversité » de l'École 
normale supérieure, organisé par Élise Demeulenaere, et les trois lecteurs anonymes ainsi que la rédaction de la revue NSS, pour leurs commentaires pertinents dont nous avons tenté de tenir compte. Nous restons, bien sûr, seules responsables des erreurs et imperfections de cet article.

\section{Références}

Barbault, R., 2006. La conservation et la gestion de la biodiversité : un défi pour l'interdisciplinarité, in Legay, J.-M. (Ed.), L'Interdisciplinarité dans les sciences de la vie, Paris, Cemagref/Cirad/Ifremer/Inra, 151-169.

Botelho, H., 2000. Fire Torch Deliverable E1 - State of the art on prescribed burning ecological effects: public concerns versus scientific results. Review of the existing research results on prescribed fire effects on Mediterranean ecosystems. a. Effects on soil, $b$. Effects on fauna, $c$. Effects on vegetation, d. Effects on trees. Fire Torch Project Contract $n^{\circ}$ ENVA-CT98-0715.

Buffière, D., Faerber, J., Le Caro, P., Métailié, J.-P., 1992. Des «écobuages » aux feux dirigés dans les Pyrénées centrales et occidentales. Évolution et rôle actuels de la pratique du débroussaillement par le feu, Revue d'analyse spatiale quantitative et appliquée, 32, 103-110.

Cernusca, A., Tappeiner, U., Bayfield, N., 1999. Land-Use Changes in European Mountain Ecosystems, Berlin, Blackwell Wissenschafts-Verlag.

Duché, Y., Rigolot, É., 2004. Équipement du territoire : le brûlage dirigé, bases scientifiques et réalisations, Rendez vous techniques, 4, 36-40.

Dumez, R., 2004. L'Herbe et le feu dans le Parc national des Cévennes : pratiques de gestion et modes de catégorisation des éleveurs et des gestionnaires. Thèse de doctorat, Muséum national d'histoire naturelle, Paris.

Génot, J.-C., Barbault, R., 2004. Quelle politique de conservation?, in Barbault, R., Chevassus-au-Louis, B., Tesseydre A. (Eds), Biodiversité et changements globaux: enjeux de société et défis pour la recherche, Paris, ADPF, 162-191.

Guisepelli, E., Fleury, P. (Eds), 2003. Paysages et agriculture dans les Alpes du Nord: représentations et aspirations de la société, Chambéry, GIS des Alpes du Nord.

Reçu le 26 août 2006. Accepté le 5 avril 2007.
Lambert, B., 1999. Gestion des milieux naturels protégés. Le brûlage : un outil au service des pasteurs et des gestionnaires, Montagnes méditerranéennes, 10, 37-39.

Larrère, C., Larrère, R., 1997. Du bon usage de la nature : pour une philosophie de l'environnement, Paris, Aubier.

Legeard, J.-P., Mallen, M., 1996. Élevage et pastoralisme : éléments d'analyse pour une politique pastorale dans le département des Alpes-Maritimes, Manosque, CERPAM.

Métailié, J.-P., 1981. Le Feu pastoral dans les Pyrénées centrales : Barousse, Oueil, Larboust, Paris, Éditions du CNRS.

Métailié, J.-P., 1998. Le savoir-brûler dans les Pyrénées. De "l'écobuage » au «brûlage dirigé », la transformation d'une pratique traditionnelle en outil de gestion de l'espace, in Rouselle, A. (Ed.), Monde rural et histoire des sciences en méditerranée : $d u$ bon sens à la logique, Perpignan, Presses Universitaires de Perpignan, 165-179.

Métailié, J.-P., 1999. Le feu : un «entre-deux» scientifique et social, Montagnes méditerranéennes, 10, 81-86.

Métailié, J.-P., Faerber, J., 2003. Quinze années de gestion des feux pastoraux dans les Pyrénées : du blocage à la concertation, Sud-Ouest européen, 16, 37-51.

Novoa, C., Dumas, S., Prodon, R., 1998. Changes in reproductive habitat of grey partridge after burning, J. Range Manage, 51, 607-613.

Ribet, N., 1996. Technique agraire ou sinistre écologique : le feu pastoral dans le Parc naturel régional des Volcans d'Auvergne, Revue d'Auvergne, 539, 111-112.

Ribet, N., 1999. L'invention du brûlage pastoral. Ethnologie d'un savoir-faire en réhabilitation, Montagnes méditerranéennes, 10, 31-36.

Ribet, N., 2000. Usages de la montagne : de la connaissance des savoirs traditionnels à l'invention d'outil de gestion. À propos des pratiques de brûlage pastoral, Montagnes méditerranéennes, 12, 158-161.

Ribet, N., 2005. La maîtrise du feu : un travail en creux qui façonne les paysages, in Le Travail et les hommes : actes du $127^{e}$ congrès du CTHS, Paris, CTHS.

Rigolot, É., 1998. Le réseau national des équipes de brûlage dirigé, Brûlages dirigés, Pastum, 51-52, 199-121.

Sigaut, F., 1975. L'Agriculture et le feu : rôle et place du feu dans les techniques de préparation du champ de l'ancienne agriculture européenne, Paris/La Haye, Mouton/ EHESS. 